\title{
RF RECOVERY USING CARA AND DEPRESSED COLLECTOR*
}

\author{
M.A. LaPointe, ${ }^{1.2}$ J.L. Hirshfield, ${ }^{1,2}$ Changbiao Wang ${ }^{2}$ \\ ${ }^{1}$ Omega-P, Inc. 345 Whitney Ave, Suite 100, New Haven, CT 06511, USA \\ ${ }^{2}$ Yale University, Beam Physics Lab, P.O. Box 208124 New Haven, CT 06520-8124, USA
}

\section{Abstract}

A system is being tested for recovery of rf power by conversion to DC pulsed power. The system consists of a cyclotron autoresonance accelerator (CARA) [1,2], and a single stage depressed collector (Fig. 1). A $50 \mathrm{kV}, 5$ A electron beam is injected into CARA, and up to $1 \mathrm{MW}$ of S-band rf power is absorbed by the beam with efficiency exceeding $95 \%$. The beam then passes to a single stage depressed collector. RF to DC collection efficiencies of $73 \%$ are predicted but, due to collector breakdown above $125 \mathrm{kV}$, a maximum collector efficiency of only $41 \%$ has been observed to date.

\section{INTRODUCTION}

The device described here is designed to recover excess if power and convert it into DC pulsed power. Applications include power recovery from spent high energy beams (e.g. FEL sources, electron linacs) that are sent through a cavity structure to regenerate RF power. The resultant RF would then be absorbed in CARA to accelerate an electron beam. The accelerated beam is sent to a single stage depressed collector for recovery. For this application CARA is acting as a transformer converting a high voltage, low current beam in to a lower voltage, higher current beam suitable for energy recovery in a depressed collector. CARA is an attractive candidate for this application on account of its high efficiency [v].

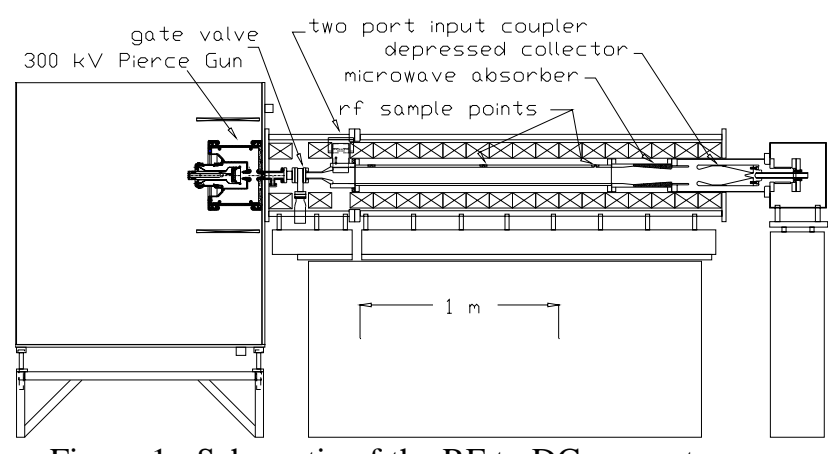

Figure 1. Schematic of the RF to DC converter.

\section{DISCUSSION}

The method described here for conversion of RF to DC proceeds by first absorbing the rf onto a low voltage, high current electron beam using CARA, which has been shown to be over $95 \%$ efficient [1] at converting RF power to beam power. The beam energy is then recovered at a depressed collector, the efficiency of which is simply the depression voltage to beam voltage ratio. The overall

\footnotetext{
*Researched sponsored bu the U.S. Department of Energy
}

efficiency of the device can be expressed by the equation

$$
\eta_{R F}=\eta_{\text {coll }} \eta_{C A R A}-\left(1-\eta_{\text {coll }}\right) P_{b i} / P_{R F} \text {. }
$$

Where $\eta_{R F}, \eta_{\text {coll }}$, and $\eta_{C A R A}$ are the overall RF conversion efficiency, collector efficiency, and CARA efficiency respectively, $P_{b i}$ and $P_{R F}$ are the initial beam power and the incident RF power, respectively.

As can be seen from this equation the primary contributions to the efficiency of the system are the collector and CARA efficiencies, but with CARA efficiencies in excess of $95 \%$ the collector efficiency dominates. The second term introduces a reduction in efficiency due to the use of a beam as the intermediary.

It is evident that one can minimize this reduction by either of two means. First, if the collector efficiency can approach $100 \%$, the second term is negligible. However this requires the depression voltage to be very near the beam voltage. Any variation of, or spread in, the energy of the beam will cause some of the beam to be reflected from the collector and subsequently reduce the efficiency.

The second means of reducing the second term is to minimize the ratio of beam to RF power. It is assumed that the amount of RF power will be a fixed quantity determined by factors outside of this device, so that the beam power must then be reduced. There are two considerations that need to addressed. First, as the beam power is reduced, for the same RF power, the final beam energy is increased and, to keep the same collector efficiency, the depression voltage needs to be increased. This can lead to breakdowns and other technical issues if the voltages get too large. The second consideration is a stalling limit in CARA [3] whereby the increasing magnetic field needed to maintain the resonance condition can eventually stop and reflect the beam. This limit is reached first for low initial beam energies.

\section{APPARATUS}

Two CARA's were used for the results presented in this paper. The first, referred to as CARA-1, [1,2] was used for the testing the absorption efficiency with varying $\mathrm{RF}$ drive power. The second, CARA-2, [4] was used to test and calibrate the depressed collector. CARA-2 also was used for the RF recovery tests.

\subsection{Electron Gun}

The gun on CARA-1 is a $100 \mathrm{kV}, 1 \times 10^{-6} \mathrm{~A}-\mathrm{V}^{-3 / 2}$ Pierce type diode. It is surrounded by a 3 -axis magnetic field trim coil used to reduce stray magnetic fields at the cathode.

The gun on CARA-2 is also a $100 \mathrm{kV}$, $1 \times 10^{-6} \mathrm{~A}-\mathrm{V}^{-3 / 2}$, Pierce type diode gun, but includes a 
$200 \mathrm{kV}$ post-acceleration stage. The ratio of the cathode voltage to the intermediate anode voltage can be varied by approximately $10 \%$ to allow flexibility in the voltage/current characteristics of the output beam (i.e., the beam current is not entirely determined by the cathode voltage). To minimize the beam power during $R F$ recovery tests, the post acceleration stage was shorted which provided similar beam parameters as CARA-1.

\subsection{RF System}

The RF systems for both CARA's were similar. RF from a SLAC XK-5 klystron was sent through a 3-dB hybrid and the two a two port, $90^{\circ}$ input coupler on CARA. The input coupler launched a rotating $\mathrm{TE}_{11}$ traveling wave in a smooth wall cylindrical waveguide. The waveguide radius is tapered in both devices for reasons associated with other experiments. [1,2,4] Any RF which is not absorbed by the beam is dissipated in a $\mathrm{RF}$ absorber. The power to/from CARA is measured in the WR-284 waveguide by a dual directional coupler prior to the 3-dB hybrid. A series of pickoff probes along the cylindrical waveguide monitor the relative rf power levels with and without the beam.

\subsection{Magnetic System}

The magnetic system consists of a series of solenoid coils surrounding the CARA waveguide and enclosed by an iron flux cage. Each coil is individually computer controlled which allows tailoring of the magnetic field to match the synchronism condition for the cyclotron resonance interaction. Compensation coils (three axis for CARA-1 and axial for CARA-2) are used to reduce the effects of stray magnetic fields at the cathode.

\subsection{Depressed Collector}

The single stage depressed collector consists of a shaped hollow beam dump electrically isolated from the main body of the tube. An insulated support under the body of the collector (not shown in Fig. 1) was installed to remove stress on the electrical insulator at the end of the device. The collector is self-depressed by use of a resistor chain between the beam dump and the tube. The collected current determines the depression voltage via the IR drop along the resistor chain. This method allows the depression voltage to be changed by moving the low voltage connection on the chain to include a variable number of resistors. For the $5 \mathrm{~A}$ beam, values of the resistance were set to give depression voltage of up to 175 $\mathrm{kV}$. Although the energy "recovered" is lost as heat in the resistor chain, the resistor chain could be replaced by either a capacitor storage system or a pulse-forming network to store and recycle the collected energy.

\section{RESULTS}

\subsection{CARA}

Several issues needed to be addressed to properly evaluate this CARA/collector system. First, CARA has been demonstrated to be efficient at moderate to high RF powers. But for this experiment, a maximum of $1.2 \mathrm{MW}$ of RF power is required. Tests of the acceleration in CARA-1 with $0.28,0.81$ and $1.2 \mathrm{MW}$ of incident RF power on a moderate energy beam $(78 \mathrm{kV}, 22 \mathrm{~A})$ have shown that the absorption efficiency is still greater than 95\% (Fig. 2 for $1.2 \mathrm{MW}$ case).

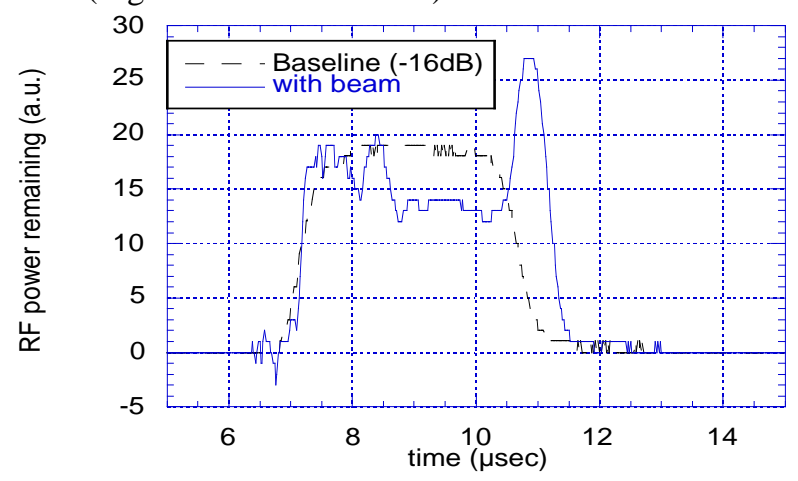

Figure 2. Sample signals at end of CARA showing $>97 \%$ of incident RF power absorbed. Baseline case is with no beam with signal externally attenuated by $16 \mathrm{~dB}$.

Another concern for a practical application of this device is the sensitivity of the optimized CARA interaction to variations in the input RF power. A test was done to optimize CARA for $1.2 \mathrm{MW}$ of input power and then to vary the RF power without changing the other settings. As seen in Fig. 3 the absorption efficiency reamined above $95 \%$ for $\pm 10 \%$ variations in the in $\mathrm{RF}$ power, and above $90 \%$ for $\pm 20 \%$ variations.

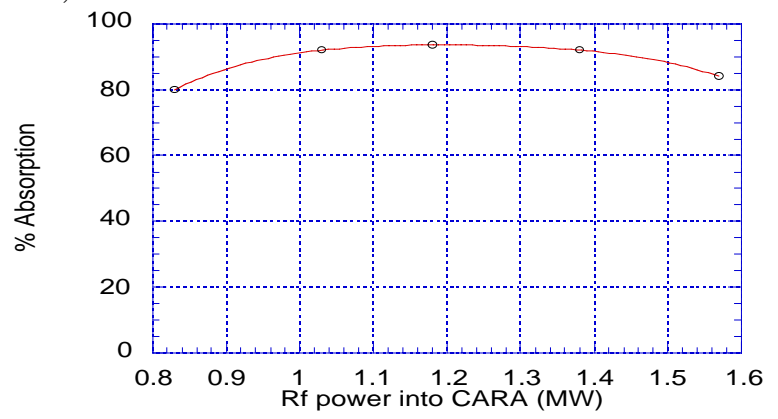

Figure 3. Variation of absorption efficiency with input RF power for optimized 1.2 MW case. All other CARA settings were unchanged.

Finally the effects of the RF power to beam power ratio and axial velocity spread on the overall interaction efficiency were simulated. Results of this simulation are shown in Table 1 for two cases. Both use $1 \mathrm{MW}$ of RF power, but two different values for the initial beam energy are used, namely $1.5 \mathrm{MW}(20 \mathrm{~A}, 74.5 \mathrm{kV})$ and $0.46 \mathrm{MW}$ $(10 \mathrm{~A}, 46.4 \mathrm{kV})$. Both cases assume a $0.5 \% \mathrm{rms}$ initial axial velocity spread. As the ratio of beam to RF power decreases, the RF conversion efficiency increases dramatically. Similar simulations show that as the rms axial velocity spread increases from $0.1 \%$ to $1 \%$ the rf conversion efficiency decreases from $70 \%$ to $35 \%$ for the 
1.5 MW beam due to the requirement of a lowered depression voltage to prevent reflection of part of the beam. For the $0.46 \mathrm{MW}$ case, the rms spread was varied from $0.4 \%$ to $1 \%$ with a decrease in $\mathrm{RF}$ conversion efficiency falling from $84 \%$ to $74 \%$.

Table 1. Simulation results for two ratios of beam power to incident RF power. Axial velocity spread was $0.5 \%$.

\begin{tabular}{|l|l|l|}
\hline & $1.5 \mathrm{MW}$ beam & $0.46 \mathrm{MW}$ beam \\
\hline$\gamma(\mathrm{kV})$ & 121 & 144 \\
\hline$\Delta \gamma(\mathrm{kV})$ & 32 & 16 \\
\hline$\eta_{\text {CARA }}$ & $92 \%$ & $98 \%$ \\
\hline Depression $(\mathrm{kV})$ & 100 & 132 \\
\hline$\eta_{\text {COLL }}$ & $50 \%$ & $85 \%$ \\
\hline
\end{tabular}

\subsection{Depressed Collector}

The depressed collector was installed on CARA-2 which was operated without RF. The electron gun was operated at beam voltages from $50-300 \mathrm{kV}$ with 5-30 A current. Several resistor setting were used and the depression voltages measured, as shown in Fig. 4. The voltages track the beam energy as expected until approximately $125 \mathrm{kV}$ is reached on the collector. At this point consistent breakdown occurs with the most probably point being the insulated support added underneath the body of the collector to remove excess stress on the main insulator. Figure 5 shows no evidence of beam loss either due to beam interception or reflection from the collector.

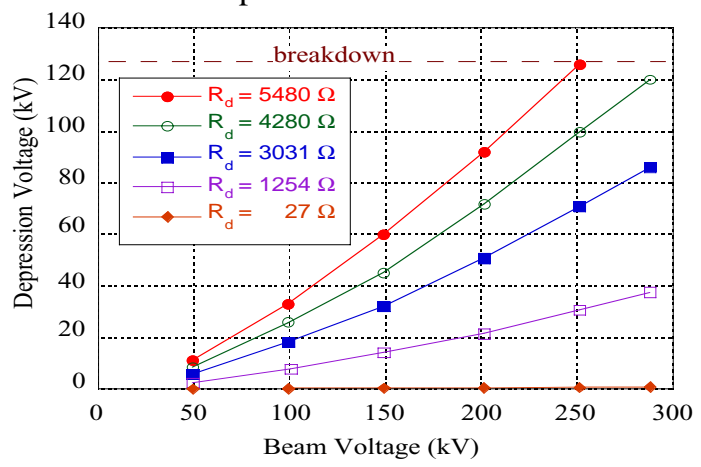

Figure 4. Deression voltage versus beam voltage for various resistor settings. Consistent breakdown ocurred at $125 \mathrm{kV}$.

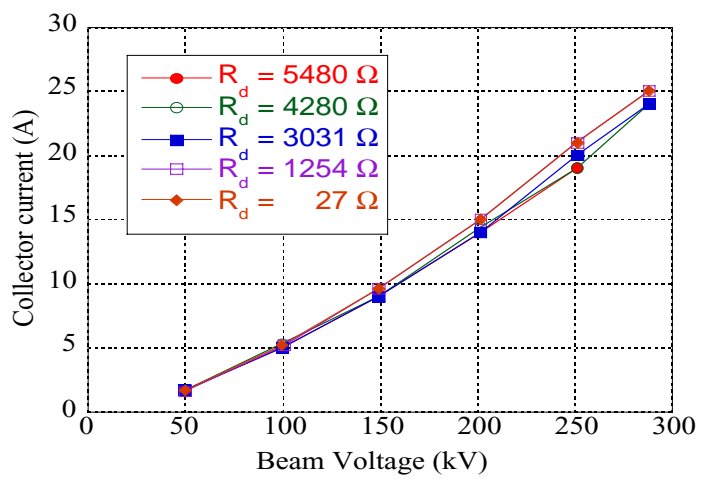

Figure 5. Measured beam current on the collector showed no evidence of current loss.

\subsection{RF to DC Conversion}

For the first full system test $84 \%$ of $1.7 \mathrm{MW}$ of RF was absorbed on a $65 \mathrm{kV}, 5 \mathrm{~A}$ beam to produce a $350 \mathrm{kV}$ beam. The measured depression voltage was only $36 \mathrm{kV}$ which gives a collector efficiency of $10 \%$ and a RF conversion efficiency of $8 \%$. The resistor chain was the same as for testing the higher beam currents. $36 \mathrm{kV}$ was the maximum depression possible with the $5 \mathrm{~A}$ beam under these circumstances. Future tests will include both higher currents and a larger resistor chain to increase the depression voltage and efficiency.

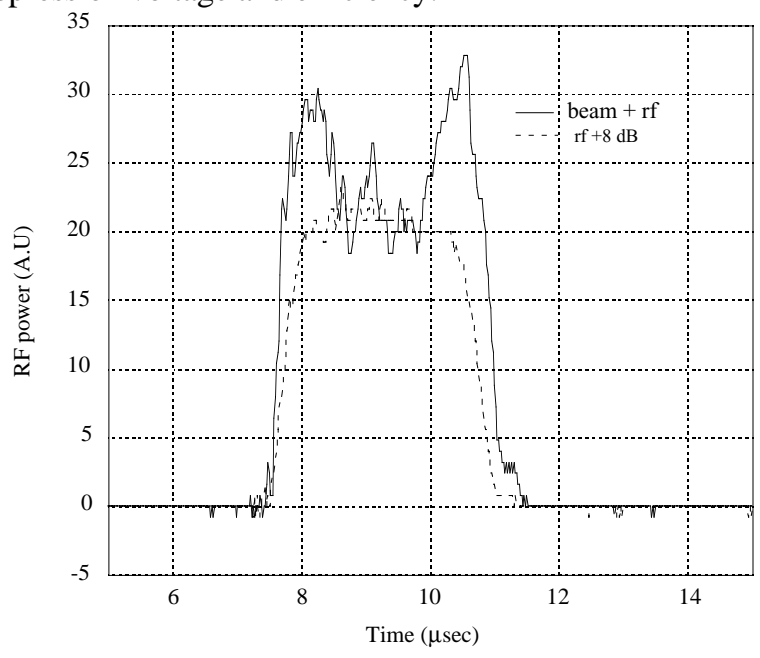

Figure 6. RF signal at end of CARA showing $84 \%$ absorption.

\section{CONCLUSION}

Simulations have shown that the combination of CARA and a depressed collector can, in principle, be an efficient means of recovering excess RF power. CARA has been shown to be relatively insensitive to modest variations in the input RF power level, and operates well with low beam and RF powers. Initial full system tests have shown the validity of the method. Depression voltages up to $125 \mathrm{kV}$ were employed. Future tests will be designed to increase the depression voltage and thus the efficiency of the device.

\section{REFERENCES}

[1] M.A. LaPointe, R.B. Yoder, Changbiao Wang, A.K. Ganguly and J.L. Hirshfield, Phys. Rev. Lett. 76, 2718 (1996)

[2] J.L. Hirshfield, M.A. LaPointe, A.K. Ganguly, R.B. Yoder and Changbiao Wang, Phys. Plasmas, 3, 2163 (1996)

[3] Changbiao Wang and J.L. Hirshfield, Phys Rev. E, 51, 2456 (1995)

[4] M.A. LaPointe, Changbiao Wang and J.L. Hirshfield, CP475, Applications of Accelerators in Research and Industry, eds. J.L. Duggan and I.L. Morgan, Woodbury, New York: AIP Press, 1999, pp. 945. 